Muro de la investigación, 2018: 3(2), agosto-diciembre,

ISSN: 2523-2886

DOI: https://doi.org/10.17162/rmi.v3i2.1157

\title{
Factores que influyen en la intención de uso del comercio móvil en los estudiantes de la Universidad Peruana Unión, durante el año 2017
}

\author{
Héctor David Mamani Ferrer ${ }^{1}$ y Alexander David De La Cruz Vargas² \\ Facultad de Ciencias Empresariales, Universidad Peruana Unión, Perú ${ }^{12}$
}

Recibido: 05 de enero de 2018

Aceptado: 08 de maro de 2018 de 2019

\section{Resumen}

La presente investigación tiene el objetivo de determinar el grado de influencia de la actitud hacia el uso, la norma subjetiva y el control conductual percibido sobre la intención de uso del comercio móvil en los estudiantes de la Universidad Peruana Unión, durante el año 2017. El desarrollo de las tecnologías de información como el internet, las redes inalámbricas y los dispositivos móviles, han dado paso al comercio móvil, definido como el conjunto de transacciones comerciales conducidas a través de dispositivos móviles usando redes inalámbricas. Su repercusión ha sido amplia a nivel mundial, aunque en Perú todavía está en crecimiento. En tal contexto, es necesario analizar los factores que motivan el uso de este tipo de actividad. La literatura propone tres factores preponderantes que afectan a la intención de uso: la actitud del usuario, determinada como una evaluación afectiva; la norma subjetiva, definida como la percepción de aprobación del entorno; y el control conductual percibido, entendido como la capacidad percibida de uso. Este estudio se ejecutó con 357 estudiantes y los datos obtenidos fueron procesados usando regresión lineal simple y múltiple. Los resultados indicaron que la actitud hacia el uso, la norma subjetiva y el control conductual percibido permiten explicar el $79.2 \%$ de la variabilidad de la intención de uso; es decir, si una persona tiene una valoración positiva del comercio móvil, presión social de su entorno y recursos necesarios para llevarlo a cabo, entonces habrá una alta probabilidad de que esté dispuesta a usarlo. Se ratificó, además, la significancia de cada factor y sus dimensiones respectivas. Por lo tanto, se concluye que la actitud hacia el uso, la norma subjetiva y el control conductual percibido influyen significativamente sobre la intención de uso del comercio móvil en los estudiantes de la Universidad Peruana Unión, durante el año 2017.

Palabras clave: Comercio móvil, intención de uso, DTPB, dispositivo móvil.

\section{Factors affecting intention to use mobile commerce in the undergraduate students of the Universidad Peruana Unión, in 2017}

\begin{abstract}
${ }^{1}$ Correspondencia al autor

E-mail: hectormamani@upeu.edu.pe
\end{abstract}

The present investigation has the objective to determine the degree of influence from attitude towards the use, subjective norm and perceived behavioral control to the intention to use mobile commerce in the undergraduate students of the Universidad Peruana Unión, in 2017. The development of information technologies such as the Internet, wireless 
networks and mobile devices, have changed the forms of commerce, giving way to mobile commerce, defined as the set of commercial transactions conducted through mobile devices using wireless networks. Its impact has been broad worldwide, but in Peru it is still growing. In this context, it is necessary to analyze the factors that motivate the use of mobile commerce. The literature proposes three important factors affecting intention to use: attitude toward use, defined as an affective evaluation; subjective norm, defined as perception of social approval; and perceived behavioral control, defined as perceived capacity of use. The study was carried out with 357 students and the data were processed by simple and multiple linear regression. The results indicated that the attitude towards the use, the subjective norm and the perceived behavioral control allow to explain the $79.2 \%$ of the variability of the intention of use. That is, if a person has a positive evaluation of the mobile commerce, social pressure of his environment and resources necessary to use it, then there will be a high probability he/she is willing to use. In addition, the significance of each factor and its respective dimensions was ratified. Therefore, it is concluded that the attitude towards the use, the subjective norm and the perceived behavioral control influences the intention to use mobile commerce in the students of the Universidad Peruana Unión, during the year 2017.

Keywords: Mobile commerce, intention to use, DTPB, mobile device.

\section{Introducción}

A lo largo de la historia, los seres humanos han intercambiado-por necesidad o deseo-bienes y servicios (Huet, 1793). Este proceso es denominado "comercio" y su finalidad es la mejora del bienestar para las partes intervinientes (Schwartz, 2001). A fin de lograrlo, el comercio ha utilizado los avances tecnológicos y en las tres últimas décadas dos innovaciones han marcado la pauta; por un lado, el internet, que ha impactado tanto a empresas como personas. En 2017, los usuarios activos de internet sobrepasaron los 3.7 mil millones a nivel global, lo que significa que al menos una de cada dos personas se conectó a la red en ese año (Ditrendia, 2017). Esto ha generado que las personas obtengan mayores capacidades y opciones a la hora de hacer compras (Zebra, 2017). Por otro lado, los dispositivos móviles se han diseminado ampliamente. Se pronosticaba que en 2017 el número de usuarios móviles a nivel mundial, habría superado los 4.9 mil millones, lo que implica que el 66\% de las personas en todo el mundo tendrían un celular (Ditrendia, 2017). En Perú, el 66\% de personas es usuario de internet y casi la mitad investiga sobre productos y servicios en línea semanalmente (Google, 2017). Asimismo, 4 de cada 10 peruanos optan conectarse a internet mediante su móvil y se espera que en 2021 sean casi 7 de cada 10 (El Comercio, 2017). Desde otro ángulo, el 73\% de peruanos se reconocen como usuarios de un smartphone, el mismo porcentaje indica realizar alguna actividad online antes de su compra (buscar información, comparar precios, explorar marcas) y en más de la mitad de los casos lo realizan mediante un dispositivo móvil (Google, 2017). 
Todo ello indica que el consumidor peruano está más informado y conectado.

En otro orden de cosas, el desarrollo de las innovaciones antes señaladas ha dado pase al desarrollo del comercio móvil, definido como el conjunto de transacciones comerciales conducidas a través de dispositivos móviles usando redes inalámbricas. Este tipo de comercio se ha incrementado considerablemente en los últimos años. Para el 2017, se estimaba que el comercio móvil representaría el 34\% del total de las ventas online en el mundo (Ditrendia, 2017), aunque existen países que ya han superado este porcentaje como Japón, Corea del Sur e Inglaterra con más del 40\% de sus transacciones online realizadas en dispositivos móviles (Criteo, 2015). En el ámbito nacional, existe una gran expectativa para el comercio móvil. En 2016, ya se había estimado que alcanzaría el 30\% del total de las transacciones electrónicas (Saavedra, 2016). Además, en un estudio patrocinado por IAB Global identificó que el 66\% de los peruanos planeaba comprar más productos o servicios en su smartphone o tablet en los siguientes seis meses, superando el promedio global que ascendía al 62\%, principalmente por motivos de ahorro de tiempo y dinero, conveniencia y protección del efectivo (Zhang \& Salomon, 2016).

A pesar de las oportunidades para el desarrollo del comercio móvil, existen factores reticentes desde el lado del consumidor: cuestiones de confianza y bajo nivel de bancarización. Respecto a lo primero, Zhang \& Salomon (2016) indican que los principales factores que restringen al consumidor de comprar más productos o servicios están asociados a cuestiones de confianza, utilidad percibida (si satisface necesidades) y facilidad de uso (es muy complicado). Con relación al índice de bancarización, esta se ha incrementado hasta $28 \%$ en los últimos seis años, pero aún es bajo comparado con otros países de la región (Chile tiene un 70\% de bancarización, Gestión 2017). Todo indica que el comercio móvil tiene un gran potencial que no se está desarrollando al máximo, aun cuando existe un ámbito favorable, por lo que es preciso determinar qué motiva al consumidor peruano a usar este tipo de comercio.

A la luz de todo este panorama contemporáneo, el objetivo de la presente investigación es determinar el grado de influencia de la actitud hacia el uso, la norma subjetiva y el control conductual percibido sobre la intención de uso del comercio móvil en los estudiantes de la Universidad Peruana Unión, durante el año 2017. 


\section{Revisión de la literatura}

El presente trabajo tiene como objeto de estudio la intención de uso del comercio móvil, por lo que una mejor comprensión del mismo demanda un análisis de los tres elementos que lo componen: tecnología móvil, comercio e intención de uso.

\section{Desarrollo tecnológico}

A fin de comprender este primer elemento, se requiere avizorar el desarrollo de las tecnologías de información, las mismas que han originado importantes cambios en la forma de vivir en todo el mundo. Servicios como el internet, las redes inalámbricas y el desarrollo de dispositivos móviles han contribuido a ello, por lo que es preciso describir su contribución.

En relación al internet, puede definirse como una red muy amplia de computadoras (Mellado, 2016). Aunque en un principio fue diseñado para uso científico y de defensa, el internet pasó a ser la mayor red de redes. Esta transición es sintetizada por Laudon \& Traver (2014) en tres etapas: innovación, institucionalización y comercialización. Uno de sus servicios más populares es la web, que funciona como un sistema de estándares universales que almacena, recupera, ordena y muestra información mediante el uso de una arquitectura cliente/servidor (Laudon \& Traver, 2014).

Respecto a las redes inalámbricas, consisten en sistemas de transmisión y recepción de datos usando redes celulares, señales de corto alcance y tecnología satelital. Respecto a lo primero, incluye las redes desarrolladas por los operadores móviles en conjunto con organismos internacionales y cuya evolución se ha dado por medio de generaciones desde la primera hasta la quinta (Manso, 2015). En relación a lo segundo, comprende las redes locales inalámbricas basadas en las señales que emiten los dispositivos en un campo reducido como Bluetooth, WiFi y NFC (Aponte \& Davila, 2011). El último abarca las redes de mayor alcance que se valen de satélites o celdas de amplia cobertura (Santomasi, 2010).

Por último, los dispositivos móviles son aparatos electrónicos de comunicación inalámbrica con interfaz de usuario, de formato pequeño y transportable (Morillo, 2017). Actualmente, existe una vasta cantidad de dispositivos móviles, sean PDAs, smartphones, tablets, etc. (Aparicio, Aguirre, \& Callejas, 2012), que usan sistemas operativos como 
Android, IPhone y Windows Mobile (este último retirado del mercado; Aponte y Davila, 2011).

\section{Evolución del comercio}

El análisis del segundo elemento, el comercio, ha estado sujeto a los cambios tecnológicos que sirvieron de soporte para nuevas formas de comercio, los más actuales basados en medios electrónicos siendo denominados como comercio electrónico y comercio móvil.

El comercio electrónico puede definirse como una transacción de valor realizada por medios electrónicos conectados a internet (Castillo \& Arroyo, 2017; Damacén, 2005). En relación a su desarrollo, se inicia en 1948 con la inserción del EDI (Electronic Data Interchange) y su posterior avance con la creación de la World Wide Web en 1992 (Gutarra \& Casanova, 2017). Luego se propaga a uso comercial acumulando-para fines de 2012 — ventas mundiales que superaron el billón de dólares (Puro Marketing, 2017). Posteriormente aparecen diversos modelos de negocio que se clasifican según la interacción entre los agentes económicos: empresa a consumidor (B2C), empresa a empresa (B2B), gobierno electrónico y consumidor a consumidor (C2C) (Laudon \& Traver, 2014). Finalmente, se puede apreciar el impacto analizando el nivel de ventas y sectores económicos involucrados. El volumen de ventas a nivel mundial tiene cifras prometedoras, y a nivel nacional el crecimiento ha sido vertiginoso. En 2015, el comercio electrónico alcanzó los 25 billones de dólares concentrando el mayor mercado en EE.UU. (7 billones), seguido de Japón (2.5 billones) y China (2 billones) (Listín Diario, 2017). En relación al volumen de ventas en Perú, en 2016 se alcanzaron los 2,300 millones de dólares, según indicó Helmut Cáceda Salazar, presidente de la Cámara Peruana de Comercio Electrónico (Capece), creciendo $198 \%$ en los últimos dos años y con una proyección de avance bianual del $30 \%$ en desktops y 55\% en dispositivos móviles (El Economista, 2017).

En relación al comercio móvil, este puede ser definido como el conjunto de transacciones comerciales conducidas a través de dispositivos móviles usando redes inalámbricas (Barnes, 2002). Este tipo de comercio presenta características únicas que la hacen una forma atractiva de hacer comercio, tales como: movilidad, localización, ubicuidad, personalización e interactividad (Cho, Kwon, \& Lee, 2007). La cadena de valor del comercio móvil envuelve varios agentes que forman parte de esta cadena, la cual culmina en la entrega del producto o servicio al cliente. Barnes (2002) señala que 
esta etapa consiste en dos áreas: infraestructura y servicios, y contenido. Por último, se establecen dos tipos de canales de acceso: según dispositivo y software. Lo primero viene de la mano con el smartphone y la tablet, y respecto al software empleado se distinguen principalmente dos canales de acceso: las aplicaciones móviles (app) y la web móvil (mobile browser; IAB, 2016).

\section{Intención de uso}

La incursión del comercio móvil y la importancia de la elaboración de una correcta estrategia de difusión dan lugar al análisis del tercer elemento, la intención de uso. Respecto al mismo se han propuesto teorías que proponen factores generales y específicos para determinar si una persona podría realizar determinado comportamiento, en este caso, usar el comercio móvil.

\section{Teoría de la acción razonada}

Esta teoría, conocida coma TRA (Theory of Reasoned Action), introduce un marco referencial para la predicción de la conducta humana por medio de tres relaciones (Fishbein y Ajzen, 1975): (1) las creencias determinan las actitudes, (2) las actitudes determinan la intención y (3) la intención determina el comportamiento. Respecto al primer tipo (1) se establecen dos líneas causales: una que vincula las creencias y las actitudes respecto al comportamiento y la otra que vincula las creencias y actitudes de naturaleza normativa. En referencia a la primera línea causal, se establece que la actitud hacia un determinado comportamiento vendría a ser la sumatoria de las creencias acerca de las consecuencias de tal comportamiento, ponderadas por la evaluación del mismo (Méndez, 2011). La segunda línea causal da paso a las creencias normativas, introduciendo la norma subjetiva como la percepción del individuo sobre la aprobación o desaprobación de determinado comportamiento por parte de las personas importantes para él (Qingfei, Shaobo, \& Gang, 2008). La segunda relación (2) establece que el peso combinado de la actitud hacia el comportamiento y de la norma subjetiva determinará la intención de llevar a cabo el comportamiento (Bauer, Reichardt, Barnes, \& Neumann, 2005). La última relación (3) establece que el inmediato antecedente de cualquier comportamiento es la intención de desarrollar el comportamiento en cuestión, por lo tanto, si la intención del individuo aumenta, tiene una mayor probabilidad de que el comportamiento sea realizado (Ajzen \& Madden, 1986). 
Esta teoría, conocida como TPB (Theory of Planned Behavior), establece la extensión de la teoría de la acción razonada e involucra el control conductual percibido como nueva variable. La TRA, que proponía a la intención como único predictor del comportamiento, resultaba ser insuficiente para predecir el comportamiento en casos en los que el sujeto no tenía control sobre tal comportamiento (Ajzen \& Madden, 1986). Por ello, se introdujo la variable "control conductual percibido" como determinante tanto de la intención como del comportamiento (Khalifa \& Shen, 2008); la misma está determinada por las creencias de control, que tienen que ver con la presencia o ausencia de ciertos recursos y oportunidades. Tales creencias pueden estar basadas en experiencias pasadas, información de segunda mano, experiencias de personas cercanas u otros factores que incrementan o reducen la dificultad para desarrollar el comportamiento en cuestión (Ajzen, 1991).

\section{Modelo de aceptación de la tecnología}

En este modelo, denominado TAM (Technology Acceptance Model), se incorpora la facilidad de uso percibida y la utilidad percibida como creencias determinantes de la aceptación de uso de la tecnología y se desarrolla como base en las relaciones de la TRA. El modelo nace con el fin de proporcionar una explicación sólida sobre los determinantes de la aceptación de sistemas de información (Maroofi, Kahrarian, \& Dehghani, 2013). Para ello, en un modelo inicial, se establecen cinco relaciones entre las variables: diseño del sistema, utilidad percibida, facilidad de uso percibida, actitud hacia el uso y uso real. En este enfoque, la actitud se deja de lado por su bajo poder de predicción y se mantiene en las ampliaciones (Wu \& Wang, 2005).

\section{Teoría de difusión de la innovación}

Esta teoría puede analizarse desde su objetivo, perspectivas y los determinantes planteados para su adopción. Respecto al primer punto, la teoría de difusión de la innovación (Innovation Diffusion Theory o IDT) fue introducida en 1962 por Rogers y su objetivo ha sido predecir la probabilidad y el nivel de adopción de una innovación según las diferentes categorías de usuarios (Kaasinen, 2005). Respecto al segundo punto esta teoría maneja dos perspectivas: el proceso de difusión y el proceso de adopción. La difusión es un macro proceso asociado a la diseminación de innovaciones desde su fuente 
hacia el público, mientras que el proceso de adopción es un micro proceso que se centra en la decisión de los individuos sobre aceptar o rechazar una innovación (Rao \& Troshani, 2007). Con relación a los determinantes del nivel de adopción de la innovación, Hoffmann (2007) menciona las cinco variables propuestas en esta teoría que afectan el nivel de adopción, concebido como la rapidez relativa en la que una innovación es adoptada por miembros de un sistema social: ventaja relativa, compatibilidad, complejidad, experimentación y visibilidad.

\section{Teoría disgregada de la conducta planificada}

La perspectiva de esta teoría propuesta por Taylor y Todd (1995) se orienta a dimensionar las variables que contempla el TPB. Este proceso, también denominado descomposición, se propone con el fin tener un mejor entendimiento sobre la relación

entre las actitudes e intenciones, y ser más manejable al revelar específicamente los predictores más relevantes que explican la adopción de tecnologías (Püschel, Afonso, \& Mauro, 2010). Para ello, los autores tomaron las creencias actitudinales, normativas y de control, planteadas como determinantes de la intención en el TPB, y generaron constructos multidimensionales a partir del análisis de las teorías existentes. Respecto a la creencias actitudinales, se analizó el TAM (Davis, 1989) y la IDT (Rogers, 1983) y se plantearon tres constructos que componen la estructura de creencias actitudinales: utilidad percibida, facilidad de uso percibida y compatibilidad.

Con relación a las creencias normativas, la teoría propone dos constructos para su estructura: influencia de similares e influencia de superiores. Se considera que los determinantes de la norma subjetiva son planteados acorde al contexto en el que se desarrolla (Pedersen, 2005). Finalmente, en relación a las creencias de control, Taylor \& Todd (1995) disgregaron el control conductual percibido tomando la autoeficacia de Bandura (1982) que estaba relacionada a la habilidad percibida y dividen los factores facilitadores de Triandis (1977) en dos dimensiones: condiciones asociadas a los recursos como tiempo o dinero y aspectos tecnológicos asociados a compatibilidad que pueden restringir el uso. 


\section{Modelo de la investigación}

Según la revisión de la literatura, se elaboró un modelo explicativo para la intención de uso, señalado en la Figura 1.

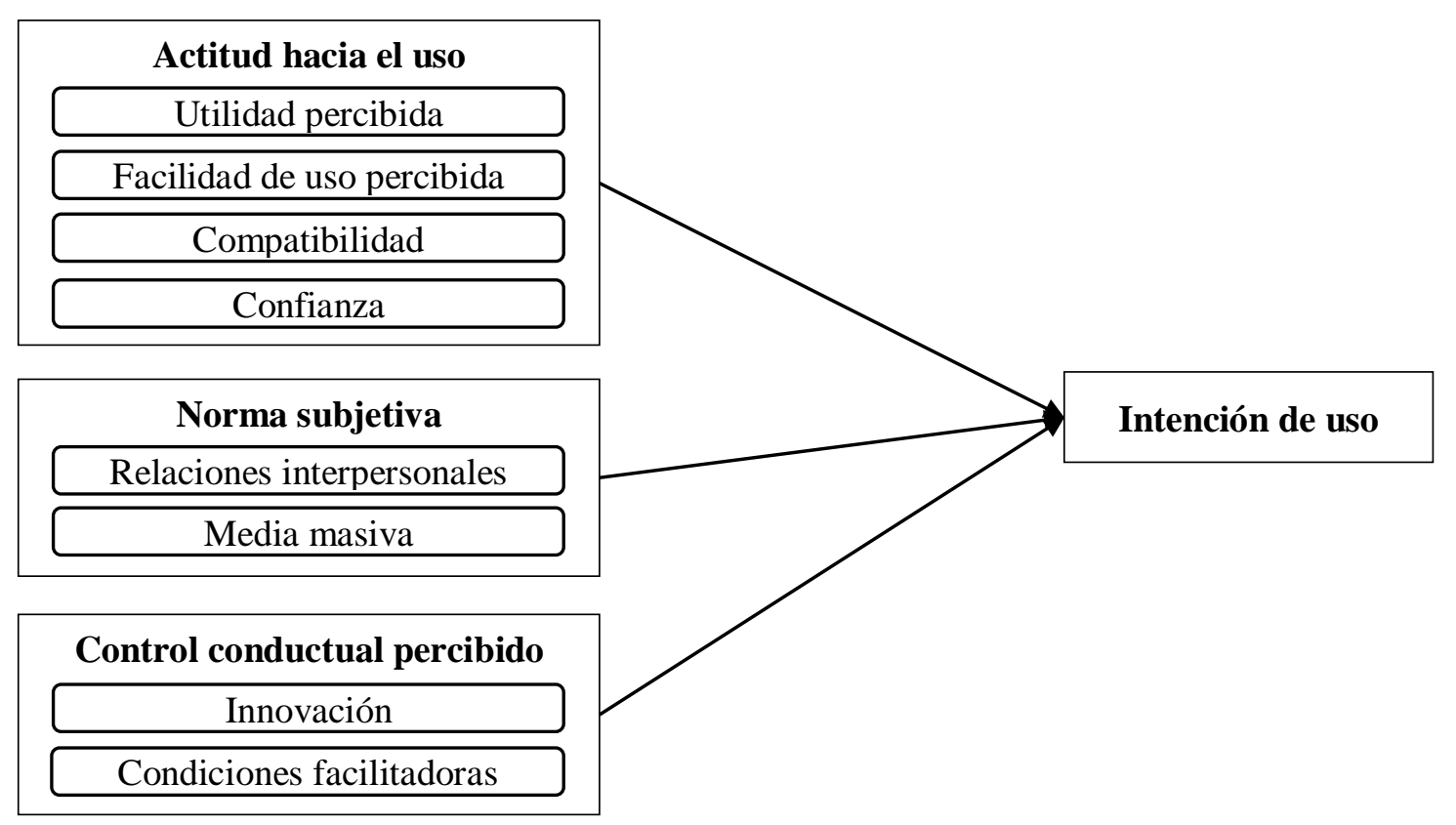

Figura 1. Modelo de la investigación. Las variables están resaltadas en negrita, enmarcadas en un rectángulo; las dimensiones se enmarcan en un rectángulo redondeado.

En base al modelo se proponen las siguientes hipótesis de investigación.

H1. La actitud hacia el uso, la norma subjetiva y el control conductual percibido influyen significativamente sobre la intención de uso del comercio móvil en los estudiantes de la Universidad Peruana Unión, durante el año 2017.

H2. La actitud hacia el uso y sus dimensiones influyen significativamente sobre la intención de uso del comercio móvil en los estudiantes de la Universidad Peruana Unión, durante el año 2017.

H3. La norma subjetiva y sus dimensiones influyen significativamente sobre la intención de uso del comercio móvil en los estudiantes de la Universidad Peruana Unión, durante el año 2017. 
H4. El control conductual percibido y sus dimensiones influyen significativamente sobre la intención de uso del comercio móvil en los estudiantes de la Universidad Peruana Unión, durante el año 2017.

\section{Materiales y métodos}

La presente investigación es de tipo explicativo, dado que busca determinar el grado de influencia entre entres variables independientes y una dependiente. Asimismo, utiliza un diseño no experimental y de corte transaccional.

\section{Participantes}

Formaron parte de la muestra 357 estudiantes pertenecientes a las cinco facultades de la Universidad Peruana Unión (Sede Lima): Ciencias Administrativas (FCE), Ciencias de la salud (FCS), Ciencias Humanas y Educación (FACIHED), Ingeniería y Arquitectura (FIA) y (FACTEO) Teología; bajo los lineamientos de un muestro estratificado.

\section{Instrumento}

Para la obtención de los datos se optó por una escala de Likert de 7 puntos adaptada de trabajos anteriores, denominada "Escala de intención de uso del comercio móvil". Esta escala estuvo compuesta de 38 ítems: 3 para la intención de uso, 19 para la actitud hacia el uso, 6 para la norma subjetiva y 10 para el control conductual percibido. Cabe indicar que para cada variable independiente se incorporaron ítems de control que ayudaron a determinar el nivel de cohesión de sus dimensiones. Para afinar la escala se realizó un análisis factorial confirmatorio y se depuraron los ítems 2, 16 y 19 dado que no se ajustaban a sus variables. Finalmente, se determinó el coeficiente de alpha de Cronbach que fue de .968 , con rangos de .730 a .946 en cada variable y dimensión.

\section{Análisis de datos}

Se aplicó una prueba piloto con 31 estudiantes de la carrera de Administración y Negocios Internacionales de la Universidad Peruana Unión, con lo que se pudo refinar la escala. Posterior a ello se ejecutó el instrumento refinado en la muestra de 357 estudiantes de la misma casa de estudios. Los datos fueron procesados en SPSS v. 22.0 utilizando estadísticos descriptivos para los datos demográficos y de uso del comercio móvil. Para 
analizar los datos de la escala, evaluar las hipótesis y la cohesión de las dimensiones se utilizó análisis de regresión lineal simple y múltiple.

\section{Resultados}

En la presente sección se presenta el análisis descriptivo del uso del comercio móvil según variables demográficas, así como las categorías de uso más demandadas. En el análisis de regresión se verifica el nivel de explicación que obtiene la actitud hacia el uso, norma subjetiva y control conductual percibido sobre la intención de uso, así como sus dimensiones.

\section{Análisis descriptivo}

Según se visualiza en la Tabla 1, más varones que mujeres han reportado haber usado el comercio móvil; asimismo, los jóvenes en la edad de 20 son más propensos a realizar este tipo de comercio. Por otro lado, es notoria la relación lineal entre el nivel socio-económico y el uso del comercio móvil. Finalmente, los estudiantes de las facultades más afines a la tecnología (ejemplo: FIA) han optado por usar el comercio móvil en mayor porcentaje que los no afines (ejemplo: FACTEO).

\section{Tabla 1}

Uso auto-reportado según sexo, edad, nivel socio-económico y facultad

\begin{tabular}{lrrrrr}
\hline & \multicolumn{4}{c}{ Uso auto-reportado } \\
\cline { 2 - 3 } \cline { 5 - 6 } Variable socio-económica & \multicolumn{2}{c}{ Uso } & & \multicolumn{2}{c}{ No uso } \\
\cline { 2 - 3 } \cline { 5 - 6 } Sexo & Frecuencia & $\%$ & & Frecuencia & $\%$ \\
Masculino & 103 & $58.9 \%$ & & 72 & $41.1 \%$ \\
Femenino & 87 & $47.8 \%$ & & 95 & $52.2 \%$ \\
& & & & & \\
Edad & 104 & $51.5 \%$ & & 98 & $48.5 \%$ \\
De 18 a 20 años & 65 & $59.1 \%$ & & 45 & $40.9 \%$ \\
De 21 a 23 años & 14 & $46.7 \%$ & & 16 & $53.3 \%$ \\
De 24 a 26 años & 6 & $75.0 \%$ & & 2 & $25.0 \%$ \\
De 27 a 29 años & 1 & $14.3 \%$ & & 6 & $85.7 \%$ \\
De 30 a más años & & & &
\end{tabular}

Nivel socio-económico

$\begin{array}{lrrrr}\text { A } & 5 & 100.0 \% & 0 & 0.0 \% \\ \text { B } & 48 & 63.2 \% & 28 & 36.8 \% \\ \text { C } & 105 & 52.5 \% & 95 & 47.5 \%\end{array}$


D

E
25

7
$44.6 \%$

$35.0 \%$
31

13
$55.4 \%$

$65.0 \%$

Facultad

FCE

36

$57.1 \%$

$42.9 \%$

FACIHED

19

$52.8 \%$

$47.2 \%$

FIA

80

$61.5 \%$

$38.5 \%$

FCS

45

$43.7 \%$

50

$56.3 \%$

FACTEO

10

$40.0 \%$

15

$60.0 \%$

Nota. FCE = Facultad de Ciencias Empresariales; FACIHED = Facultad de Ciencias Humanas y Educación; FIA = Facultad de Ingeniería y Arquitectura; FCS = Facultad de Ciencias de la Salud; FACTEO = Facultad de Teología.

En relación a las categorías que los participantes identificaron como de mayor demanda, la Tabla 2 muestra los resultados respecto a ello. Como se puede visualizar, existe una amplia preferencia por la adquisición de datos para usar las diferentes aplicaciones de los dispositivos móviles, también un porcentaje considerable de los participantes manifestaron haber realizado compras de boletos para diversos centros de entretenimientos (34\%), ropa, calzado y accesorios (33\%), así como aplicaciones de pago a través de su dispositivo móvil (30.4\%).

Tabla 2

Porcentaje de uso del comercio móvil según categoría de uso

\begin{tabular}{lcc}
\hline Categorías de uso & Frecuencia & Porcentaje \\
\hline Datos (megas) & 119 & $62.6 \%$ \\
Boletería & 65 & $34.2 \%$ \\
Moda & 62 & $32.6 \%$ \\
Aplicaciones & 57 & $30.0 \%$ \\
Entretenimiento & 55 & $28.9 \%$ \\
Turismo & 43 & $22.6 \%$ \\
Alimentación & 40 & $21.1 \%$ \\
Equipos electrónicos & 30 & $15.8 \%$ \\
Servicios públicos & 29 & $15.3 \%$ \\
Hogar & 27 & $14.2 \%$ \\
Salud y belleza & 27 & $14.2 \%$ \\
Transporte & 25 & $13.2 \%$ \\
\hline Nota. Las respuestas por &
\end{tabular}

Nota. Las respuestas por cada participante podían ser múltiples, por lo que la sumatoria de frecuencias puede ser igual o superior a la cantidad de la muestra.

\section{Análisis de regresión}


Los resultados de los análisis de regresión para la evaluación de cada hipótesis de la investigación son interpretados a continuación. Respecto a la hipótesis 1 (H1), la Tabla 3 muestra que se justifica la incursión de las tres variables independientes dado que aportan de forma significativa a la explicación de la variabilidad de la intención de uso, siendo que en conjunto puede explicar el $79.2 \%$ de la misma. La Tabla 4 señala los coeficientes de la ecuación de regresión indicando que tanto la actitud hacia el uso y el control conductual percibido tienen mayor influencia que la norma subjetiva y son similares entre ambas.

Tabla 3

Predicción de la variabilidad de la intención de uso, según modelo de investigación

\begin{tabular}{ccccccc}
\hline & & & & Error típ. de & \multicolumn{2}{c}{ Estadísticos de cambio } \\
\cline { 5 - 7 } Paso & $R$ & $R^{2}$ & $R^{2}$ corregida & estimación & Cambio en $R^{2}$ & Cambio en $F$ \\
\hline $1^{\text {a }}$ & .823 & .677 & .676 & .73039 & .677 & $744.432^{* * *}$ \\
$2^{\text {b }}$ & .876 & .767 & .765 & .62183 & .090 & $135.771^{* * *}$ \\
$3^{\text {c }}$ & .891 & .794 & .792 & .58562 & .027 & $46.134^{* * *}$ \\
\hline
\end{tabular}

Nota. Las distancias entre la $R^{2}$ y la $R^{2}$ corregida no son amplias por lo que el tamaño muestral y la cantidad de variables independientes no están sesgando la variabilidad. Método utilizado: Pasos sucesivos. ${ }^{\text {a}}$ Variable independiente: Actitud hacia el uso. ${ }^{b}$ Variable independiente: Actitud hacia el uso y Control conductual percibido. ${ }^{c}$ Variable independiente: Actitud hacia el uso, Control conductual percibido y Norma subjetiva. $* * * p<.001$

Tabla 4

Coeficientes de regresión parcial del modelo de la investigación

\begin{tabular}{lcccc}
\hline \multirow{2}{*}{ Variables independientes } & \multicolumn{3}{l}{ Coeficientes no estandarizados ${ }^{\mathrm{a}}$} \\
\cline { 2 - 5 } & $B[\mathrm{IC}]$ & $\begin{array}{c}\text { Error } \\
\text { típ. }\end{array}$ & $\begin{array}{c}\text { Coeficientes } \\
\text { tipificados }\end{array}$ & $t$ \\
\hline (Constante) & $-0.582[-0.87,-0,30]$ & 0.144 & & $-4.041^{* * * *}$ \\
Actitud hacia el uso & $0.444[0.35,0.54]$ & 0.050 & 0.364 & $8.927^{* * *}$ \\
Control conductual & $0.432[0.34,0.52]$ & 0.045 & 0.364 & $9.589^{* * *}$ \\
percibido & $0.287[0.20,0.37]$ & 0.042 & 0.257 & $6.792^{* * *}$ \\
\hline Norma subjetiva &
\end{tabular}

Nota . IC = Intervalo de confianza al 95\% [límite inferior, límite superior]; error típ. = Error típico de estimación. ${ }^{a}$ Estos coeficientes determinan el modelo de regresión. ${ }^{\mathrm{b}}$ Evalúa el peso de cada regresor sin el efecto de la escala en el que es medido. $* * * p<.001$ 
Respecto a la hipótesis 2 (H2), la Tabla 5 muestra que la actitud hacia el uso puede explicar el $67.6 \%$ de la variabilidad de la intención de uso, bajo una relación lineal significativa. Además, como se visualiza en la Tabla 6, cada una de sus dimensiones explica más de la mitad de la variabilidad de la intención de uso, a excepción de la confianza (42.9\%), también bajo relaciones lineal significativas.

Tabla 5

Predicción de la variabilidad de la intención de uso, según la actitud hacia el uso

\begin{tabular}{cccccc}
\hline & & \multicolumn{3}{c}{ Error típ. de } \\
Variable & $R$ & $R^{2}$ & $R^{2}$ corregida & estimación & $F$ \\
independiente & $R$ & .677 & .676 & .73039 & $744.432 * * *$ \\
\hline Actitud hacia el uso & .823 & &
\end{tabular}

a dependiente: Intención de uso.

$* * * p<.001$

Tabla 6

Predicción de la variabilidad de la intención de uso, según dimensiones de la actitud hacia el uso

\begin{tabular}{|c|c|c|c|c|c|c|}
\hline \multirow[b]{2}{*}{ Dimensión } & \multicolumn{3}{|c|}{ Varianza explicada } & \multirow[b]{2}{*}{ ANOVA $^{\mathrm{a}}$} & \multicolumn{2}{|c|}{$\begin{array}{l}\text { Coeficiente de } \\
\text { regresión }\end{array}$} \\
\hline & $R$ & $R^{2}$ & $\begin{array}{c}R^{2} \\
\text { corregida }\end{array}$ & & $B$ & $t$ \\
\hline Utilidad percibida & $\begin{array}{c}.80 \\
3\end{array}$ & $\begin{array}{c}.64 \\
5\end{array}$ & .644 & $\begin{array}{c}645.594 * * \\
*\end{array}$ & 0.917 & $25.409 * * *$ \\
\hline (Constante) & & & & & 0.564 & $3.444 * * *$ \\
\hline Compatibilidad & $\begin{array}{c}.75 \\
3\end{array}$ & $\begin{array}{l}.56 \\
7\end{array}$ & .566 & $\begin{array}{c}464.726 * * \\
*\end{array}$ & 0.800 & $21.558 * * *$ \\
\hline (Constante) & & & & & 1.174 & $7.112 * * *$ \\
\hline $\begin{array}{l}\text { Facilidad de uso } \\
\text { percibida }\end{array}$ & $\begin{array}{c}.73 \\
6\end{array}$ & $\begin{array}{c}.54 \\
2\end{array}$ & .541 & $\begin{array}{c}419.781 * * \\
*\end{array}$ & 0.854 & $20.489 * * *$ \\
\hline (Constante) & & & & & 0.795 & $4.160 * * *$ \\
\hline Confianza & $\begin{array}{c}.65 \\
6\end{array}$ & $\begin{array}{c}.43 \\
0\end{array}$ & .429 & $\begin{array}{c}267.997 * * \\
*\end{array}$ & 0.713 & $16.371 * * *$ \\
\hline (Constante) & & & & & 1.583 & $8.284 * * *$ \\
\hline
\end{tabular}

Nota. Las dimensiones son introducidas por separado como variables independientes respecto a la intención de uso para determinar su influencia de forma aislada (sin el efecto de las otras dimensiones y variables). 
${ }^{a}$ El contraste de regresión se efectúa con los valores $F$ para cada dimensión por separado.

$* * * p<.001$

La cohesión de las dimensiones se aprecia en la Tabla 7, y se puede apreciar que todas las dimensiones de la actitud hacia el uso explican el $71.5 \%$ de los indicadores actitudinales, que miden a la variable de forma general, en especial las dimensiones provenientes del TAM (utilidad percibida y facilidad de uso percibida). También se aprecia que la constante es dejada de lado en la ecuación de regresión por lo que hay suficiencia de las dimensiones para pertenecer a la variable.

\section{Tabla 7}

Cohesión de las dimensiones de la actitud hacia el uso

\begin{tabular}{|c|c|c|c|c|c|c|}
\hline \multirow[b]{2}{*}{ Variable independiente $\mathrm{e}^{\mathrm{a}}$} & \multicolumn{3}{|c|}{ Varianza explicada } & \multirow[b]{2}{*}{$A N O V A^{b}$} & \multicolumn{2}{|c|}{$\begin{array}{l}\text { Coeficientes de } \\
\text { regresión }\end{array}$} \\
\hline & $R$ & $R^{2}$ & $\begin{array}{c}R^{2} \\
\text { corregida }\end{array}$ & & $B$ & $t$ \\
\hline Compatibilidad & $\begin{array}{c}0.84 \\
8\end{array}$ & $\begin{array}{c}0.71 \\
8\end{array}$ & 0.715 & $\begin{array}{c}224.364 * * \\
*\end{array}$ & 0.317 & $5.476^{*}$ \\
\hline Utilidad percibida & & & & & 0.311 & $\begin{array}{l}4.869 * * \\
*\end{array}$ \\
\hline $\begin{array}{l}\text { Facilidad de uso } \\
\text { percibida }\end{array}$ & & & & & 0.248 & $\begin{array}{l}4.306^{* *} \\
*\end{array}$ \\
\hline Confianza & & & & & 0.100 & $2.248^{*}$ \\
\hline (Constante) & & & & & 0.278 & 1.838 \\
\hline
\end{tabular}

Nota. Se determinó la influencia de las dimensiones sobre indicadores que medían exclusivamente a la actitud hacia el uso como un todo para validar la cohesión de las dimensiones.

${ }^{\text {a }}$ Variable dependiente $=$ Actitud hacia el uso (indicadores actitudinales); las dimensiones son introducidas como variables independientes en un solo modelo de regresión.

${ }^{\mathrm{b}}$ El contraste de regresión se efectúa con los valores $F$ para el modelo de regresión. $* p<.05 . * * * p<.001$

En relación a la hipótesis 3, la Tabla 8 indica que la norma subjetiva es capaz de explicar el $60.2 \%$ de la variabilidad de la intención de uso, bajo una relación lineal significativa $(F=538.546, p>.05)$. Por otro lado, sus dimensiones, de forma independiente, también explican un porcentaje moderado de la variabilidad de la intención de uso, con relativa mayor importancia en las relaciones interpersonales (Tabla $9)$. 
Tabla 8.

Predicción de la variabilidad de la intención de uso, según la norma subjetiva

\begin{tabular}{cccccc}
\hline $\begin{array}{c}\text { Variable } \\
\text { independiente }\end{array}$ & $R$ & $R^{2}$ & $R^{2}$ corregida & $\begin{array}{c}\text { Error típ. de } \\
\text { la estimación }\end{array}$ & $F$ \\
\hline Norma subjetiva & .776 & .603 & .602 & 0.81018 & $538.546^{* * *}$ \\
\hline Nota. Variabe
\end{tabular}

Nota. Variable dependiente: Intención de uso.

$* * * p<.001$

Tabla 9

Predicción de la variabilidad de la intención de uso, según dimensiones de la norma subjetiva

\begin{tabular}{|c|c|c|c|c|c|c|}
\hline \multirow[b]{2}{*}{ Dimensión } & \multicolumn{3}{|c|}{ Varianza explicada } & \multirow[b]{2}{*}{ ANOVA $^{\mathrm{a}}$} & \multicolumn{2}{|c|}{ Coeficiente de regresión } \\
\hline & $R$ & $R^{2}$ & $R^{2}$ corregida & & $B$ & $t$ \\
\hline $\begin{array}{l}\text { Relaciones } \\
\text { interpersonales }\end{array}$ & .728 & .530 & .529 & $400.383 * * *$ & 0.746 & $20.010 * * *$ \\
\hline (Constante) & & & & & 1.363 & $8.100 * * *$ \\
\hline Media masiva & .651 & .424 & .423 & $261.654 * * *$ & 0.646 & $16.176 * * *$ \\
\hline (Constante) & & & & & 1.549 & $7.930 * * *$ \\
\hline
\end{tabular}

Nota. Las dimensiones son introducidas por separado como variables independientes respecto a la intención de uso para determinar su influencia de forma aislada (sin el efecto de las otras dimensiones y variables).

${ }^{a} E l$ contraste de regresión se efectúa con los valores $F$ para cada dimensión por separado.

$* * * p<.001$

Las dimensiones de la norma subjetiva, según se visualiza en la Tabla 10, están cohesionadas al guardar un $71 \%$ de porcentaje de explicación respecto a los indicadores normativos que evalúan a la variable de forma general, además existe suficiencia de variables al dejar de lado a la constante en la ecuación de regresión.

Tabla 10

Cohesión de las dimensiones de la norma subjetiva

\begin{tabular}{|c|c|c|c|c|c|c|}
\hline \multirow{2}{*}{$\begin{array}{c}\text { Variable } \\
\text { independiente }\end{array}$} & \multicolumn{3}{|c|}{ Varianza explicada } & \multirow[b]{2}{*}{ ANOVA $^{\mathrm{b}}$} & \multicolumn{2}{|c|}{ Coeficiente de regresión } \\
\hline & $R$ & $R^{2}$ & $R^{2}$ corregida & & $B$ & $t$ \\
\hline $\begin{array}{l}\text { Relaciones } \\
\text { interpersonales }\end{array}$ & .843 & .711 & .710 & $436.132 * * *$ & 0.738 & $18.871 * * *$ \\
\hline Media masiva & & & & & 0.157 & $4.153 * * *$ \\
\hline (Constante) & & & & & 0.275 & 1.886 \\
\hline
\end{tabular}


Nota. Se determinó la influencia de las dimensiones sobre indicadores que medían exclusivamente a la norma subjetiva como un todo para validar la cohesión de las dimensiones.

${ }^{\text {a }}$ Variable dependiente $=$ Norma subjetiva (indicadores normativos); las dimensiones son introducidas como variables independientes en un solo modelo de regresión.

${ }^{\mathrm{b}} \mathrm{El}$ contraste de regresión se efectúa con los valores $F$ para el modelo de regresión. $* * * p<.001$

Finalmente, con relación a la hipótesis 4 (H4), la Tabla 11 deja ver que el control conductual percibido tiene la capacidad de explicar el $65.6 \%$ de la variabilidad de la intención de uso y su relación es lineal con un nivel crítico menor a .05. Además, cada una de sus dimensiones pueden explicar más del $50 \%$ de la variabilidad de la intención de uso (Tabla 12).

Tabla 11

Predicción de la variabilidad de la intención de uso, según el control conductual percibido

\begin{tabular}{ccccccc}
\hline & & \multicolumn{4}{c}{ Error típ. } \\
de la \\
Variable independiente & $R$ & $R^{2}$ & $R^{2}$ corregida & estimación & $F$ \\
\hline Control conductual percibido & .811 & .657 & .656 & .75239 & $681.084 * * *$
\end{tabular}

Nota. Variable dependiente: Intención de uso.

$* * * p<.001$

Tabla 12

Predicción de la variabilidad de la intención de uso, según dimensiones de la norma subjetiva

\begin{tabular}{lcccccc}
\hline & \multicolumn{3}{c}{ Varianza explicada } & & \multicolumn{2}{c}{ Coeficiente de regresión } \\
\cline { 2 - 5 } \cline { 5 - 7 } Dimensión & $R$ & $R^{2}$ & $R^{2}$ corregida & ANOVA $^{\mathrm{a}}$ & $B$ & $t$ \\
\hline Innovación & .744 & .553 & .552 & $439.720^{* * *}$ & 0.822 & $20.97^{* * *}$ \\
$\quad($ Constante) & & & & & 0.696 & $3.635^{* * *}$ \\
& & & & & & \\
$\begin{array}{l}\text { Condiciones } \\
\text { facilitadoras }\end{array}$ & .777 & .603 & .602 & $540.247^{* * *}$ & 0.863 & $23.243^{* * *}$ \\
$\quad($ Constante) & & & & & 0.813 & $7.930^{* * *}$ \\
\hline
\end{tabular}

Nota. Las dimensiones son introducidas por separado como variables independientes respecto a la intención de uso para determinar su influencia de forma aislada (sin el efecto de las otras dimensiones y variables).

${ }^{a}$ El contraste de regresión se efectúa con los valores $F$ para cada dimensión por separado.

$* * * p<.001$ 
Las dimensiones del control conductual percibido, como se ve en la Tabla 13, son capaces de explicar el 66\% de la variabilidad de los indicadores de control, que miden a la variable en general, por lo que hay cohesión de las dimensiones. Además, son suficientes para explicar la variable dado que se dejó de lado a la constante.

Tabla 13

Cohesión de las dimensiones del control conductual percibido

\begin{tabular}{|c|c|c|c|c|c|c|}
\hline \multirow{2}{*}{$\begin{array}{c}\text { Variable } \\
\text { independiente }\end{array}$} & \multicolumn{3}{|c|}{ Varianza explicada } & \multirow[b]{2}{*}{ ANOVA } & \multicolumn{2}{|c|}{ Coeficiente de regresión } \\
\hline & $R$ & $R^{2}$ & $R^{2}$ corregida & & $B$ & $t$ \\
\hline $\begin{array}{l}\text { Condiciones } \\
\text { facilitadoras }\end{array}$ & .814 & .662 & .660 & $346.697 * * *$ & 0.779 & $14.864 * * *$ \\
\hline Innovación & & & & & 0.160 & $3.064 * *$ \\
\hline (Constante) & & & & & 0.292 & 1.690 \\
\hline
\end{tabular}

Nota. Se determinó la influencia de las dimensiones sobre indicadores que medían exclusivamente al control conductual percibido como un todo para validar la cohesión de las dimensiones.

${ }^{\text {a }}$ Variable dependiente $=$ Control conductual percibido (indicadores de control); las dimensiones son introducidas como variables independientes en un solo modelo de regresión.

${ }^{b}$ El contraste de regresión se efectúa con los valores $F$ para el modelo de regresión. $* * p<.01 . * * * p<.001$

\section{Discusión}

La presente tesis propuso un modelo conceptual para la intención de uso del comercio móvil que integró a la TAM, IDT, TPB, DTPB y el factor confianza y fue evaluado bajo dos enfoques: el primero basado en los lineamientos de la TPB y el segundo basado en la disgregación de la DTPB, aunque por medio de dimensiones y no como variables independientes.

El primer enfoque fue la base de la hipótesis 1 (H1), y los resultados dados validan dicho enfoque, por lo que se podría aseverar que una persona estará dispuesta a usar el comercio móvil si se cumplen tres condiciones: (1) realiza una positiva valoración del uso del comercio móvil, (2) recibe presión social de su entorno a favor de este tipo de comercio y (3) tiene los recursos y conocimientos a su disposición para efectuarlo. Esto ocurre en un entorno en el que la persona toma sus decisiones de forma racional, según explica Fishbein y Azjen (1975). Las creencias que tenga sobre determinado 
comportamiento tendrán incidencia sobre la intención y posterior ejecución del comportamiento. Estudios anteriores han tenido resultados similares como es el caso de Pedersen (2005) y Khalifa y Cheng (2002), que evidenciaron empíricamente que estas tres variables tenían influencia sobre la intención de uso del comercio móvil. No obstante, las limitantes asociadas al tipo de población sobre el que se ejecutó la investigación y el conocimiento actual de las personas sobre el comercio móvil deben ser superadas en estudios posteriores.

El segundo enfoque determinó las hipótesis 2, 3 y 4 (H2, H3, H4). Con relación a la actitud hacia el uso (H2), se ratificó la influencia sobre la intención de uso por parte de la misma y de sus dimensiones de forma independiente. Esto indica que, si una persona obtiene una valoración positiva del uso del comercio móvil, producto de haber percibido que es útil para su vida diaria (utilidad percibida), es sencillo de manejar (facilidad de uso percibida), se amolda a su estilo de vida (compatibilidad) y no tiene problemas de seguridad y privacidad (confianza), habrá una mayor probabilidad de que esté predispuesta a usarlo (intención de uso). Estudios asociados al comercio móvil han presentado resultados similares con ratificando una relación directa entre la actitud hacia el uso y la intención de uso (Brown, Venkatesh, \& Hoehle, 2014; Chew, 2006; Fishbein \& Ajzen, 1975).

Con relación a la norma subjetiva, los resultados fueron similares que con la anterior variable, por lo que se afirmaría que si una persona percibe que los referentes de su entorno cercano (amigos, padres, etc.) y los medios de comunicación con los que interactúa aprueban el uso del comercio móvil, entonces estará bajo presión social y lo predispondrá a usarlo. Este resultado está acorde a lo señalado por Maslow (1943) quien explicaba que la persona es un ser sociable y con necesidades de aceptación e integración en el grupo social en el que se desenvuelve. Es por eso que Rogers (1983) aseveraba que al incursionar una innovación las personas estarían atentas a los referentes, que definen lo aceptable o no en una sociedad. Este resultado ha sido similar al de otros estudios que han tomado en cuenta la presión social (Bhatti, 2007; Chew, 2006; Taylor \& Todd, 1995), así también es un constructo clave en la UTAUT (en sus diferentes ampliaciones) y en las variaciones del TAM.

Para terminar, el control conductual percibido ha sido validado como antecedente de la intención de uso, lo que se traduce en que una persona con más recursos y conocimientos, y que además esté abierta a nuevas ideas, podrá estar en mejores condiciones para poder usar el comercio móvil y estando a su alcance, lo usará. 
Investigaciones anteriores han ratificado también la influencia del control conductual percibido sobre la intención de uso (Bhatti, 2007; Püschel et al., 2010), pero también con un efecto significativo en el uso real (Ajzen \& Madden, 1986; Taylor \& Todd, 1995) dado que existen situaciones en las que no solo basta con estar predispuesto, sino que se requieren recursos tangibles e intangibles para llevar a cabo un comportamiento. Esta lineal causal debe ser estudiada más a fondo, dado que los modelos más recientes sobre adopción de tecnologías (por ejemplo: UTAUT) eliminan la relación causal con la intención de uso y validan solo el uso real. No obstante, para la presente investigación, el moderado conocimiento sobre el comercio móvil no permite medir el uso real, pero sí la intención de uso.

\section{Conclusiones}

La presente investigación fue elaborada con el fin de determinar el grado de influencia de la actitud hacia el uso, la norma subjetiva y el control conductual percibido sobre la intención de uso del comercio móvil en los estudiantes de la Universidad Peruana Unión, durante el año 2017. Con los resultados obtenidos y su contraste con estudios anteriores se llegan a las siguientes conclusiones.

La actitud hacia el uso, la norma subjetiva y el control conductual influyen significativamente en la intención de uso del comercio móvil en los sujetos de estudio, logrando predecir gran parte de la variabilidad de la intención de uso. Además, la actitud hacia el uso y el control conductual percibido tienen una mayor relevancia que la norma subjetiva.

La actitud hacia el uso y sus dimensiones influyen significativamente en la intención de uso del comercio móvil en los sujetos de estudio. Por lo tanto, la evaluación por parte de los usuarios potenciales del valor agregado que tiene la implementación del comercio móvil estará sujeta a la utilidad que ellos perciban, a su facilidad de manejo y aprendizaje, a la relación que guarden con sus valores, necesidades y experiencias, y a los mecanismos de seguridad y protección de la privacidad que se empleen.

La norma subjetiva y sus dimensiones influyen significativamente en la intención de uso del comercio móvil en los sujetos de estudio. Es por ello que la difusión de tecnologías asociadas al comercio móvil debe estar enfocada en las personas referentes, a fin de que puedan repercutir su experiencia y motivar a las personas a usar este tipo de tecnología. 
El control conductual percibido y sus dimensiones influyen significativamente en la intención de uso del comercio móvil en los sujetos de estudio. Tanto las condiciones facilitadoras y la innovación se ajustan adecuadamente como dimensiones del control conductual percibido y sirven como base para diferenciar entre la cantidad de recursos y conocimiento disponibles para usar el comercio móvil y la capacidad de adquirirlos.

\section{Referencias}

Ajzen, I. (1991). The theory of planned behavior. Organizational Behavior and Human Decision Processes, 50, 179-211.

Ajzen, I., \& Madden, T. J. (1986). Prediction of goal-directed behavior: Attitudes, intentions, and perceived behavioral control. Journal of Experimental Social Psychology, 22(5), 453-474.

Aparicio, J., Aguirre, C., \& Callejas, E. (2012). Tecnología móvil como herramienta de apoyo en la educación media. Recuperado a partir de http://www.utec.edu.sv/media/investigaciones/files/6.Tecnologiamovilcomounahe rramientadeapoyo.pdf

Aponte, S., \& Davila, C. (2011). Sistemas Operativos Moviles: Funcionalidades, Efectividad y Aplicaciones Utiles en Colombia (Tesis de licenciatura). Universidad EAN, Bogotá, Colombia.

Barnes, S. J. (2002). The Mobile Commerce Value Chain: Analysis and Future Developments. International Journal of Information Management, 22, 91-108.

Bauer, H. H., Reichardt, T., Barnes, S. J., \& Neumann, M. M. (2005). Driving consumer acceptance of Mobile Marketing: A theorical framework and empirical study. Journal of Electronic Commerce Research, 6(3), 181-192.

Bhatti, T. (2007). Exploring Factors Influencing the Adoption of Mobile Commerce. Journal of Internet Banking and Commerce, 12(3), 1-13.

Brown, S., Venkatesh, V., \& Hoehle, H. (2014). Technology Adoption Decisions in the Household: A Seven-Model Comparison. Journal of the Association for Information Science and Technology, 66(9), 1933-1949.

Castillo, A., \& Arroyo, D. (2017). Retos y estrategias para el crecimiento del comercio electrónico peruano, en el modelo de negocio B2C, tomando como referencia al país de Chile (Tesis de licenciatura). Universidad Peruana de Ciencias Aplicadas, Lima, Perú. 
Chew, A. (2006). The Adoption of M-Commerce in the United States (Tesis de licenciatura). California State University, California, Estados Unidos.

Cho, D., Kwon, H. J., \& Lee, H. (2007). Analysis of trust in Internet and Mobile Commerce Adoption. En R. Sprague (Presidencia), Proceedings of the 40th Hawaii International Conference on System Sciences. Hawaii, Estados Unidos.

Criteo. (2015). State of Mobile Commerce. Growing like a weed. Madrid, España: Criteo. Damacén, D. (2005). El comercio electrónico en las negociaciones comerciales de las PYMES en el Perú (Tesis de maestría). Universidad Nacional Mayor de San Marcos, Lima, Perú.

Davis, F. (1986). A Technology Acceptance Model for Empirically Testing New End-User Information Systems: Theory and Results (Tesis de doctorado). Massachusetts Institute of Technology, Massachusetts, Estados Unidos.

Davis, F. (1989). Perceived Usefulness, Perceived Ease of Use, and User Acceptance of Information Technology. MIS Quarterly, 13(3), 319-340.

Davis, F. (1993). User acceptance of information technology: system characteristics, user perceptions and behavioral impacts. International Journal of Man-Machine Studies, 38(3), 475-487.

Ditrendia. (2017). Informe Mobile en España y en el Mundo 2017. Madrid, España: Ditrendia.

El Comercio. (2017, junio 15). PwC: penetración de internet móvil será 67,8\% en el 2021. El Comercio. Recuperado el 19 de octubre de 2017, a partir de www.elcomercio.pe El Economista. (2017, marzo 31). Comercio electrónico en Perú. El Economista. Recuperado el 9 de agosto de 2017, a partir de http://www.eleconomistaamerica.pe/economia-eAmperu/noticias/8263017/03/17/Comercio-electronico-en-Peru-facturo-2800millones-de-dolares/

Fishbein, M., \& Ajzen, I. (1975). Belief, Attitude, Intention, and Behavior: An Introduction to Theory and Research. MA: Addison-Wesley.

Gestión. (2015, febrero 24). Crecimiento de comercio móvil triplicará al de e-commerce a nivel mundial. Gestión. Recuperado el 24 de mayo de 2016, a partir de http://gestion.pe/tecnologia/crecimiento-comercio-movil-triplicara-al-commercenivel-mundial-2124395 
Google. (2017, agosto 1). Perú conectado: consumidores más informados a la hora de comprar. Recuperado el 19 de octubre de 2017, a partir de www.thinkwithgoogle.com

Gutarra, L., \& Casanova, G. (2017). El valor estratégico percibido y la adopción del comercio electrónico en las empresas importadoras de polietileno de baja densidad de Lima y Callao (Tesis de licenciatura). Universidad San Ignacio de Loyola, Lima, Perú.

Hoffmann, V. (2007). Diffusion of Innovations. Knowledge and Innovation Management. Stuttgart, Alemania: Hohenheim University.

Huet, P. (1793). Historia del comercio y de la navegación de los antiguos. Recuperado a partir de https://books.google.com.pe/books?hl=es\&lr=\&id=MJOLhRHnTOsC\&oi=fnd\&pg $=$ PA1\&dq=historia+del+comercio\&ots=EN20A0dPN7\&sig=bWwmbCrchkxE3ue EmqzVcIRbGgU\&redir_esc $=\mathrm{y} \# \mathrm{v}=$ onepage $\& \mathrm{q}=$ historia del comercio $\& \mathrm{f}=$ false

IAB. (2016). A Global Perspective of Mobile Commerce. New York, Estados Unidos: IAB.

Kaasinen, E. (2005). User acceptance of mobile services - value, ease of use, trust and ease of adoption. Helsinki, Finlandia: VTT Publications.

Khalifa, M., \& Cheng, S. (2002). Adoption of Mobile Commerce: Role of Exposure. En R. Sprague (Presidencia), Proceedings of the 35th Hawaii International Conference on System Sciences. Hawaii, Estados Unidos.

Khalifa, M., \& Shen, K. N. (2008). Drivers for Transactional B2C M-Commerce Adoption: Extended Theory of Planned Behavior. Journal of Computer Information Systems, 48(3), 111-117.

Laudon, K., \& Traver, C. (2014). E-commerce 2014. Business, technology, society (10ma Ed.). New Jersey, Estados Unidos: Pearson Education.

Listín Diario. (2017, abril 22). El comercio electrónico mueve en el mundo 25 billones de dólares. Listín Diario. Recuperado el 9 de agosto de 2017, a partir de http://www.listindiario.com/las-mundiales/2017/04/21/462776/el-comercioelectronico-mueve-en-el-mundo-25-billones-de-dolares

Manso, F. (2015). Análisis de Modelos de Negocios Basados en Big Data para Operadores Móviles (Tesis de maestría). Universidad de San Andrés, Buenos Aires, Argentina. 
Maroofi, F., Kahrarian, F., \& Dehghani, M. (2013). An Investigation of Initial Trust in Mobile Banking. International Journal of Academic Research in Business and Social Sciences, 3(9), 394-403.

Maslow, A. (1943). A theory of human motivation. Psychological Review, 50(4), 370396.

Mellado, A. (2016). La infraestructura de telecomunicaciones y el desarrollo económico de los países (Tesis de maestría). Universidad del Pacífico, Lima, Perú.

Méndez, F. (2011). Desarrollo de una escala para la medición de la ubicuidad en el marco de m-comercio (Tesis de doctorado). Universidad Autónoma de Madrid, Madrid, España.

Pedersen, E. (2005). Adoption of mobile Internet services: An exploratory study of mobile commerce early adopters. Journal of organizational computing and electronic commerce, 15(3), 203-222.

Puro Marketing. (2017, mayo 16). Breve historia y momentos claves en la evolución del comercio electrónico. Puro Marketing. Recuperado el 13 de agosto de 2017, a partir de http://www.puromarketing.com/76/22158/breve-historia-momentos-clavesevolucion-comercio-electronico.html

Püschel, J., Afonso, J., \& Mauro, J. (2010). Mobile banking: Proposition of an integrated adoption intention framework. International Journal of Bank Marketing, 28(5), 389-409.

Qingfei, M., Shaobo, J., \& Gang, Q. (2008). Mobile Commerce User Acceptance Study in China: A Revised UTAUT Model. Tsinghua Science and Technology, 13(3), 257-264.

Rao, S., \& Troshani, I. (2007). A Conceptual Framework and Propositions for the Acceptance of Mobile Services. Journal of Theoretical and Applied Electronic Commerce Research, 2(2), 61-73.

Rogers, E. (1983). Diffusion of Innovations (3ra Ed.). New York, Estados Unidos: The Free Press.

Saavedra, M. (2016, marzo 29). E-commerce a través de móviles se duplicaría al cierre del año. El Comercio. Recuperado el 24 de mayo de 2016, a partir de https://elcomercio.pe/economia/peru/e-commerce-traves-moviles-duplicariacierre-ano-213922

Schwartz, P. (2001). El comercio internacional en la historia del pensamiento económico. Documento de trabajo (Vol. 3). Madrid, España: IUDEM. 
Taylor, S., \& Todd, P. (1995). Understanding Information Technology Usage: A test of Competing Models. Information Systems Research, 6(2), 144-176.

Venkatesh, V., \& Davis, F. (1996). A model of the antecedents of perceived ease of use: Development and test. Decision Sciences, 27(3), 451-481.

Wu, J., \& Wang, S. (2005). What drives mobile commerce? An empirical evaluation of the revised technology acceptance model. Information \& Management, 42, 719729.

Zebra. (2017). Redefiniendo retail: Estudio sobre la industria de comercio minorista 2017. Illinois, Estados Unidos: Zebra Technologies. 